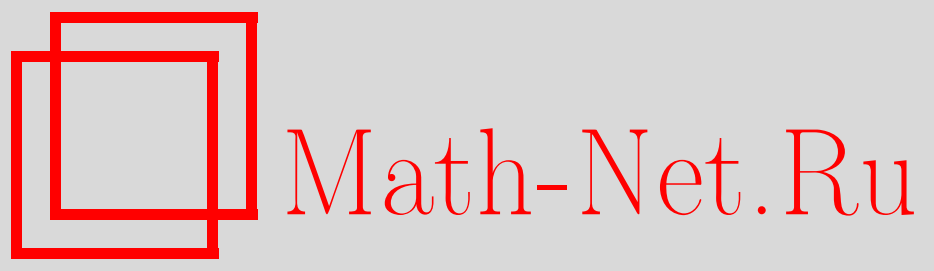

А. В. Латышев, А. А. Юшканов, Поперечная электрическая проводимость квантовой столкновительной плазмы в подходе Мермина, ТМФ, 2013, том 175, номер 1, 132143

DOI: https://doi.org/10.4213/tmf8422

Использование Общероссийского математического портала Math-Net.Ru подразумевает, что вы прочитали и согласны с пользовательским соглашением http://www . mathnet.ru/rus/agreement

Параметры загрузки:

IP : 54.205 .225 .156

26 апреля 2023 г., $17: 52: 27$

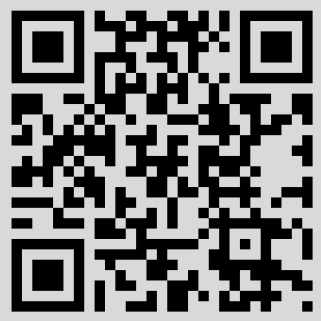




\title{
ПОПЕРЕЧНАЯ ЭЛЕКТРИЧЕСКАЯ ПРОВОДИМОСТЬ КВАНТОВОЙ СТОЛКНОВИТЕЛЬНОЙ ПЛАЗМЫ В ПОДХОДЕ МЕРМИНА
}

\begin{abstract}
Выведены формулы для поперечной электрической проводимости и диэлектрической проницаемости в квантовой столкновительной плазме. Используется кинетическое уравнение для матрицы плотности в релаксационном приближении в пространстве импульсов. Показано, что, когда постоянная Планка стремится к нулю, выведенная формула переходит в классическую, а когда стремится к нулю частота столкновений электронов (т. е. плазма переходит в бесстолкновительную), выведенные формулы переходят в полученные ранее Линдхардом. Показано также, что, когда волновое число стремится к нулю, квантовая проводимость переходит в классическую. Проведено сравнение выведенной проводимости с проводимостью по Линдхарду и с классической проводимостью.
\end{abstract}

Ключевые слова: Линдхард, Мермин, квантовая столкновительная плазма, проводимость, проницаемость, матрица плотности, коммутатор, вырожденная плазма.

DOI: $10.4213 / \operatorname{tmf} 8422$

\section{1. ВВЕДЕНИЕ}

В хорошо известной работе Мермина [1] на основе анализа неравновесной матрицы плотности в $\tau$-приближении было получено выражение для продольной диэлектрической проницаемости квантовой столкновительной плазмы. Ранее в работе Линдхарда [2] были получены выражения для продольной и поперечной диэлектрических проницаемостей квантовой бесстолкновительной плазмы. Затем Кливер и Фукс показали [3], что прямое обобщение формул Линдхарда на случай столкновительной плазмы (путем замены $\omega \rightarrow \omega+i / \tau)$ некорректно. Этот недостаток для продольной диэлектрической проницаемости был преодолен в работе [1]. До настоящего времени не получено корректного выражения для поперечной диэлектрической проницаемости в случае вырожденной квантовой столкновительной плазмы. Цель настоящей работы - восполнить этот пробел.

Свойства электрической проводимости и диэлектрической проницаемости, рассчитанных по формулам Линдхарда [2], подробно изучались в монографии [4].

* Московский государственный областной университет, Москва, Россия.

E-mail: avlatyshev@mail.ru,yushkanov@inbox.ru 
Поперечная диэлектрическая проницаемость необходима для описания оптических свойств металлов, в частности для описания скин-эффекта [5]-[7] и поверхностных плазменных колебаний [8], [9]. Диэлектрическая проницаемость также необходима для описания энергетических потерь заряженных частиц в твердых телах [10]. В настоящее время растет интерес к изучению различных свойств квантовой плазмы (см, например, работы [11]-[17]).

\section{2. КИНЕТИЧЕСКОЕ УРАВНЕНИЕ ДЛЯ МАТРИЦЫ ПЛОТНОСТИ}

Пусть векторный потенциал электромагнитного поля является гармоническим, т. е. изменяется как $\mathbf{A}=\mathbf{A}(\mathbf{r}) e^{-i \omega t}$. Мы рассматриваем поперечную проводимость, поэтому выполняется соотношение $\operatorname{div} \mathbf{A}(\mathbf{r}, t)=0$. Связь между векторным потенциалом и напряженностью электрического поля дается следующим выражением: $\mathbf{A}(\mathbf{q})=-(i c / \omega) \mathbf{E}(\mathbf{q})$. Равновесная матрица плотности имеет вид

$$
\tilde{\rho}=\left(1+e^{(H-\mu) / k_{\mathrm{B}} T}\right)^{-1},
$$

где $T$ - температура плазмы, $k_{\mathrm{B}}$ - постоянная Больцмана, $\mu$ - химический потенциал плазмы, $H$ - гамильтониан. В линейном приближении гамильтониан записывается как

$$
H=\frac{(\mathbf{p}-(e / c) \mathbf{A})^{2}}{2 m}=\frac{\mathbf{p}^{2}}{2 m}-\frac{e}{2 m c}(\mathbf{p} \mathbf{A}+\mathbf{A p}),
$$

где $\mathbf{p}$ - оператор импульса, $\mathbf{p}=-i \hbar \nabla$, постоянные $e$ и $m$ - заряд и масса электрона, $c$ - скорость света. Мы можем представить этот гамильтониан в виде суммы двух операторов: $H=H_{0}+H_{1}$, где

$$
H_{0}=\frac{\mathbf{p}^{2}}{2 m}, \quad H_{1}=-\frac{e}{2 m c}(\mathbf{p} \mathbf{A}+\mathbf{A} \mathbf{p}) .
$$

Рассмотрим кинетическое уравнение [1] для матрицы плотности Шредингера с интегралом столкновений в форме $\tau$-приближения:

$$
i \hbar \frac{\partial \rho}{\partial t}=[H, \rho]+\frac{i \hbar}{\tau}(\tilde{\rho}-\rho)
$$

где $\nu=1 / \tau$ - эффективная частота столкновений частиц плазмы, $\tau$ - характерное время между двумя последовательными столкновениями, $\hbar$ - постоянная Планка, $[H, \rho]=H \rho-\rho H-$ коммутатор, $\tilde{\rho}$ - равновесная матрица плотности. В работе [18] показано, что частота столкновений $\nu$ может зависеть от частоты колебаний поля $\omega$.

В линейном приближении по внешнему полю мы ищем матрицу плотности в виде

$$
\rho=\tilde{\rho}^{(0)}+\rho^{(1)}
$$

где $\rho^{(1)}$ - поправка (возмущение) к равновесной матрице плотности, обусловленная наличием электромагнитного поля, $\tilde{\rho}^{(0)}$ - равновесная матрица плотности, отвечающая "равновесному" оператору Гамильтона $H_{0}$. Представим равновесную матрицу плотности $\tilde{\rho}(2.1)$ в виде

$$
\tilde{\rho}=\tilde{\rho}^{(0)}+\tilde{\rho}^{(1)}
$$


и рассмотрим коммутатор $[H, \tilde{\rho}]$. В линейном приближении этот коммутатор равен

$$
[H, \tilde{\rho}]=\left[H_{0}, \tilde{\rho}^{(1)}\right]+\left[H_{1}, \tilde{\rho}^{(0)}\right]
$$

и $[H, \tilde{\rho}]=0$. Для коммутаторов из правой части равенства $(2.5)$ мы находим

$$
\begin{aligned}
\left\langle\mathbf{k}_{1}\left|\left[H_{0}, \tilde{\rho}^{(1)}\right]\right| \mathbf{k}_{2}\right\rangle & =\left(E_{\mathbf{k}_{1}}-E_{\mathbf{k}_{2}}\right) \tilde{\rho}^{(1)}\left(\mathbf{k}_{1}-\mathbf{k}_{2}\right), \\
\left\langle\mathbf{k}_{1}\left|\left[H_{1}, \tilde{\rho}^{(0)}\right]\right| \mathbf{k}_{2}\right\rangle & =\left(f_{\mathbf{k}_{1}}-f_{\mathbf{k}_{2}}\right)\left\langle\mathbf{k}_{1}\left|H_{1}\right| \mathbf{k}_{2}\right\rangle= \\
& =\frac{e}{2 m c}\left(f_{\mathbf{k}_{1}}-f_{\mathbf{k}_{2}}\right)\left(\mathbf{k}_{1}+\mathbf{k}_{2}\right) \mathbf{A}\left(\mathbf{k}_{1}-\mathbf{k}_{2}\right),
\end{aligned}
$$

где

$$
f_{\mathbf{k}}=\left(1+e^{\left(E_{\mathbf{k}}-\mu\right) / k_{\mathrm{B}} T}\right)^{-1}, \quad E_{\mathbf{k}}=\frac{\hbar^{2} \mathbf{k}^{2}}{2 m}, \quad \mathbf{p}=\hbar \mathbf{k} .
$$

Из соотношений (2.4)-(2.6) вытекает, что

$$
\tilde{\rho}^{(1)}\left(\mathbf{k}_{1}-\mathbf{k}_{2}\right)=-\frac{e \hbar}{2 m c} \frac{f_{\mathbf{k}_{1}}-f_{\mathbf{k}_{2}}}{E_{\mathbf{k}_{1}}-E_{\mathbf{k}_{2}}}\left(\mathbf{k}_{1}+\mathbf{k}_{2}\right) \mathbf{A}\left(\mathbf{k}_{1}-\mathbf{k}_{2}\right) .
$$

С помощью соотношений (2.3)-(2.5) мы линеаризуем кинетическое уравнение (2.2). Получаем следующее уравнение:

$$
i \hbar \frac{\partial \rho^{(1)}}{\partial t}=\left[H_{0}, \rho^{(1)}\right]+\left[H_{1}, \tilde{\rho}^{(0)}\right]+i \hbar \nu\left(\tilde{\rho}^{(1)}-\rho^{(1)}\right) .
$$

Заметим, что возмущение $\rho^{(1)} \sim e^{-i \omega t}$, таким образом, уравнение (2.8) принимает вид $(\hbar \omega+i \hbar \nu) \rho^{(1)}=\left[H_{0}, \rho^{(1)}\right]+\left[H_{1}, \tilde{\rho}^{(0)}\right]+i \hbar \nu \tilde{\rho}^{(1)}$. Отсюда следует, что

$$
(\hbar \omega+i \hbar \nu)\left\langle\mathbf{k}_{1}\left|\rho^{(1)}\right| \mathbf{k}_{2}\right\rangle=\left\langle\mathbf{k}_{1}\left|\left[H_{0}, \rho^{(1)}\right]\right| \mathbf{k}_{2}\right\rangle+\left\langle\mathbf{k}_{1}\left|\left[H_{1}, \tilde{\rho}^{(0)}\right]\right| \mathbf{k}_{2}\right\rangle+i \hbar \nu\left\langle\mathbf{k}_{1}\left|\tilde{\rho}^{(0)}\right| \mathbf{k}_{2}\right\rangle .
$$

Здесь

$$
\left\langle\mathbf{k}_{1}\left|\left[H_{0}, \rho^{(1)}\right]\right| \mathbf{k}_{2}\right\rangle=\left(E_{\mathbf{k}_{1}}-E_{\mathbf{k}_{2}}\right)\left\langle\mathbf{k}_{1}\left|\rho^{(1)}\right| \mathbf{k}_{2}\right\rangle=\left(E_{\mathbf{k}_{1}}-E_{\mathbf{k}_{2}}\right) \rho^{(1)}\left(\mathbf{k}_{1}-\mathbf{k}_{2}\right) .
$$

Преобразуем равенство (2.9) к виду

$$
\begin{aligned}
(\hbar \omega+i \hbar \nu & \left.-E_{\mathbf{k}_{1}}+E_{\mathbf{k}_{2}}\right) \rho^{(1)}\left(\mathbf{k}_{1}-\mathbf{k}_{2}\right)= \\
& =\frac{e \hbar}{2 m c}\left(f_{\mathbf{k}_{1}}-f_{\mathbf{k}_{2}}\right)\left(\mathbf{k}_{1}+\mathbf{k}_{2}\right) \mathbf{A}\left(\mathbf{k}_{1}-\mathbf{k}_{2}\right)+i \hbar \nu \tilde{\rho}^{(1)}\left(\mathbf{k}_{1}-\mathbf{k}_{2}\right) .
\end{aligned}
$$

Последнее слагаемое в этом равенстве заменим с учетом (2.7). Из полученного уравнения находим

$$
\rho^{(1)}\left(\mathbf{k}_{1}-\mathbf{k}_{2}\right)=\frac{e \hbar}{2 m c} \Xi\left(\mathbf{k}_{1}, \mathbf{k}_{2}\right)\left[f_{\mathbf{k}_{1}}-f_{\mathbf{k}_{2}}\right]\left(\mathbf{k}_{1}+\mathbf{k}_{2}\right) \mathbf{A}\left(\mathbf{k}_{1}-\mathbf{k}_{2}\right),
$$

где

$$
\Xi\left(\mathbf{k}_{1}, \mathbf{k}_{2}\right)=\frac{E_{\mathbf{k}_{1}}-E_{\mathbf{k}_{2}}-i \hbar \nu}{\left(E_{\mathbf{k}_{1}}-E_{\mathbf{k}_{2}}\right)\left(\hbar \omega+i \hbar \nu-E_{\mathbf{k}_{1}}+E_{\mathbf{k}_{2}}\right)} .
$$

В уравнении (2.10) мы положим $\mathbf{k}_{1}=\mathbf{k}, \mathbf{k}_{2}=\mathbf{k}-\mathbf{q}$, тогда

$$
\left\langle\mathbf{k}_{1}\left|\rho^{(1)}\right| \mathbf{k}_{2}\right\rangle=\left\langle\mathbf{k}\left|\rho^{(1)}\right| \mathbf{k}-\mathbf{q}\right\rangle=\rho^{(1)}(\mathbf{q})=-\frac{e \hbar}{m c} \Xi(\mathbf{k}, \mathbf{q})\left[f_{\mathbf{k}}-f_{\mathbf{k}-\mathbf{q}}\right] \mathbf{k} \mathbf{A}(\mathbf{q}),
$$

где

$$
\Xi(\mathbf{k}, \mathbf{q})=\frac{E_{\mathbf{k}}-E_{\mathbf{k}-\mathbf{q}}-i \hbar \nu}{\left(E_{\mathbf{k}}-E_{\mathbf{k}-\mathbf{q}}\right)\left(\hbar \omega+i \hbar \nu-E_{\mathbf{k}}+E_{\mathbf{k}-\mathbf{q}}\right)} .
$$




\section{3. ПЛОТНОСТЬ ТОКА}

Плотность тока $\mathbf{j}=\mathbf{j}(\mathbf{q}, \omega, \nu)$ определяется как

$$
\mathbf{j}=\frac{e}{8 \pi^{3} m} \int\left\langle\mathbf{k}+\frac{\mathbf{q}}{2}\left|\left(\mathbf{p}-\frac{e}{c} \mathbf{A}\right) \rho+\rho\left(\mathbf{p}-\frac{e}{c} \mathbf{A}\right)\right| \mathbf{k}-\frac{\mathbf{q}}{2}\right\rangle d \mathbf{k}
$$

Подставим (2.3) в интеграл и заметим, что

$$
\begin{gathered}
\left\langle\mathbf{k}+\frac{\mathbf{q}}{2}\left|\mathbf{p} \rho^{(1)}+\rho^{(1)} \mathbf{p}\right| \mathbf{k}-\frac{\mathbf{q}}{2}\right\rangle=2 \hbar \mathbf{k} \rho^{(0)}(\mathbf{q}), \\
\left\langle\mathbf{k}+\frac{\mathbf{q}}{2}\left|\mathbf{A} \tilde{\rho}^{(0)}+\tilde{\rho}^{(0)} \mathbf{A}\right| \mathbf{k}-\frac{\mathbf{q}}{2}\right\rangle=\mathbf{A}(\mathbf{q})\left[\tilde{\rho}^{(0)}\left(\mathbf{k}+\frac{\mathbf{q}}{2}\right)+\tilde{\rho}^{(0)}\left(\mathbf{k}-\frac{\mathbf{q}}{2}\right)\right], \\
\frac{1}{8 \pi^{3}} \int \tilde{\rho}^{(0)}\left(\mathbf{k}+\frac{\mathbf{q}}{2}\right) d \mathbf{k}=\frac{1}{8 \pi^{3}} \int \tilde{\rho}^{(0)}\left(\mathbf{k}-\frac{\mathbf{q}}{2}\right) d \mathbf{k}=\frac{N}{2},
\end{gathered}
$$

где $N$ - числовая плотность (концентрация) плазмы. Следовательно, плотность тока равна

$$
\mathbf{j}=-\frac{e^{2} N}{m c} \mathbf{A}(\mathbf{q})+\frac{e \hbar}{4 \pi^{3} m} \int\left\langle\mathbf{k}+\frac{\mathbf{q}}{2}\left|\rho^{(1)}\right| \mathbf{k}-\frac{\mathbf{q}}{2}\right\rangle \mathbf{k} d \mathbf{k}
$$

здесь первое слагаемое есть не что иное, как калибровочная плотность тока. С помощью очевидной замены переменных в интеграле из (3.2) это соотношение можно преобразовать к виду

$$
\mathbf{j}=-\frac{e^{2} N}{m c} \mathbf{A}(\mathbf{q})+\frac{e \hbar}{4 \pi^{3} m} \int\left\langle\mathbf{k}\left|\rho^{(1)}\right| \mathbf{k}-\mathbf{q}\right\rangle \mathbf{k} d \mathbf{k} .
$$

Подставляя (2.11) в подынтегральное выражение в (3.3), получаем следующее выражение для плотности тока:

$$
\mathbf{j}=-\frac{e^{2} N}{m c} \mathbf{A}(\mathbf{q})-\frac{e^{2} \hbar^{2}}{m^{2} c} \frac{1}{4 \pi^{3}} \int[\mathbf{k} \mathbf{A}(\mathbf{q})] \Xi(\mathbf{k}, \mathbf{q})\left(f_{\mathbf{k}}-f_{\mathbf{k}-\mathbf{q}}\right) \mathbf{k} d \mathbf{k} .
$$

Направим ось $x$ вдоль вектора $\mathbf{q}$, а ось $y$ - вдоль вектора $\mathbf{A}$. Тогда предыдущее векторное равенство (3.4) может быть переписано в виде трех скалярных:

$$
\begin{aligned}
& j_{y}=-\frac{e^{2} N}{m c} A(\mathbf{q})-\frac{e^{2} \hbar^{2} A(\mathbf{q})}{m^{2} c} \frac{1}{4 \pi^{3}} \int \Xi(\mathbf{k}, \mathbf{q})\left(f_{\mathbf{k}}-f_{\mathbf{k}-\mathbf{q}}\right) k_{y}^{2} d \mathbf{k}, \\
& j_{x}=j_{z}=0 .
\end{aligned}
$$

Нетрудно видеть, что выражение для плотности тока можно представить в следующей инвариантной форме:

$$
\mathbf{j}=-\mathbf{A}(\mathbf{q})\left(\frac{N e^{2}}{m c}+\frac{e^{2} \hbar^{2}}{8 \pi^{3} m^{2} c} \int \Xi(\mathbf{k}, \mathbf{q})\left(f_{\mathbf{k}}-f_{\mathbf{k}-\mathbf{q}}\right) \mathbf{k}_{\perp}^{2} d \mathbf{k}\right), \quad \mathbf{k}_{\perp}^{2}=\mathbf{k}^{2}-\left(\frac{\mathbf{k q}}{q}\right)^{2} .
$$

Разлагая $\Xi(\mathbf{k}, \mathbf{q})$ на дроби, представим плотность тока в виде

$$
\begin{aligned}
\mathbf{j}(\mathbf{q}, \omega, \nu)= & -\mathbf{A}(\mathbf{q})\left(\frac{N e^{2}}{m c}+\frac{e^{2} \hbar^{2}}{8 \pi^{3} m^{2} c} \int \frac{f_{\mathbf{k}}-f_{\mathbf{k}-\mathbf{q}}}{E_{\mathbf{k}}-E_{\mathbf{k}-\mathbf{q}}} \mathbf{k}_{\perp}^{2} d \mathbf{k}+\right. \\
& \left.+\frac{e^{2} \hbar^{3} \omega}{8 \pi^{3} m^{2} c} \int \frac{f_{\mathbf{k}}-f_{\mathbf{k}-\mathbf{q}}}{\left(E_{\mathbf{k}}-E_{\mathbf{k}-\mathbf{q}}\right)\left[E_{\mathbf{k}}-E_{\mathbf{k}-\mathbf{q}}-\hbar(\omega+i \nu)\right]} \mathbf{k}_{\perp}^{2} d \mathbf{k}\right) .
\end{aligned}
$$


Первые два члена в этом соотношении не зависят от частоты $\omega$ и определяются диссипативными свойствами материала, которые зависят от частоты столкновений $\nu$. Эти члены являются универсальными параметрами, определяющими диамагнетизм Ландау.

\section{4. ПОПЕРЕЧНАЯ ПРОВОДИМОСТЬ И ПРОНИЦАЕМОСТЬ}

Учитывая связь векторного потенциала с напряженностью электромагнитного поля, а также связь плотности тока с электрическим полем, на основании соотношения (3.5) получаем следующее выражение для поперечной электрической проводимости $\sigma_{\mathrm{tr}}(\mathbf{q}, \omega, \nu)$ :

$$
\frac{\sigma_{\mathrm{tr}}}{\sigma_{0}}=\frac{i \nu}{\omega}\left(1+\frac{\hbar^{2}}{8 \pi^{3} m N} \int \Xi(\mathbf{k}, \mathbf{q})\left(f_{\mathbf{k}}-f_{\mathbf{k}-\mathbf{q}}\right) \mathbf{k}_{\perp}^{2} d \mathbf{k}\right)
$$

где $\sigma_{0}=e^{2} N / m \nu$ - статическая проводимость. С учетом этого равенства напишем выражение для поперечной диэлектрической проницаемости:

$$
\varepsilon_{\mathrm{tr}}=1-\frac{\omega_{\mathrm{p}}^{2}}{\omega^{2}}\left(1+\frac{\hbar^{2}}{8 \pi^{3} m N} \int \Xi(\mathbf{k}, \mathbf{q})\left(f_{\mathbf{k}}-f_{\mathbf{k}-\mathbf{q}}\right) \mathbf{k}_{\perp}^{2} d \mathbf{k}\right)
$$

где $\omega_{\mathrm{p}}$ - плазменная (ленгмюровская) частота, $\omega_{\mathrm{p}}^{2}=4 \pi e^{2} N / m$. Отсюда видно, что выполняется одно из соотношений, называемое правилом $f$-сумм (см., например, работы [4], [19], [20]) для поперечной диэлектрической проницаемости. Это правило выражается формулой (4.200) из монографии [19]:

$$
\int_{-\infty}^{\infty} \varepsilon_{\operatorname{tr}}(\mathbf{q}, \omega, \nu) \omega d \omega=\pi \omega_{\mathrm{p}}^{2}
$$

Разлагая $\Xi(\mathbf{k}, \mathbf{q})$ на элементарные дроби, для поперечной электрической проводимости и диэлектрической проницаемости имеем следующие явные представления:

$$
\frac{\sigma_{\mathrm{tr}}}{\sigma_{0}}=\frac{i \nu}{\omega}\left(1+\frac{\omega J_{\omega}+i \nu J_{\nu}}{\omega+i \nu}\right), \quad \varepsilon_{\mathrm{tr}}=1-\frac{\omega_{\mathrm{p}}^{2}}{\omega^{2}}\left(1+\frac{\omega J_{\omega}+i \nu J_{\nu}}{\omega+i \nu}\right),
$$

где

$$
J_{\nu}=\frac{\hbar^{2}}{8 \pi^{3} m N} \int \frac{f_{\mathbf{k}}-f_{\mathbf{k}-\mathbf{q}}}{E_{\mathbf{k}}-E_{\mathbf{k}-\mathbf{q}}} \mathbf{k}_{\perp}^{2} d \mathbf{k}, \quad J_{\omega}=\frac{\hbar^{2}}{8 \pi^{3} m N} \int \frac{f_{\mathbf{k}}-f_{\mathbf{k}-\mathbf{q}}}{E_{\mathbf{k}}-E_{\mathbf{k}-\mathbf{q}}-\hbar(\omega+i \nu)} \mathbf{k}_{\perp}^{2} d \mathbf{k} .
$$

Вместо вектора $\mathbf{k}$ введем безразмерный вектор $\mathbf{K}=\mathbf{k} / k_{\mathrm{F}}$, где $k_{\mathrm{F}}=p_{\mathrm{F}} / \hbar-$ волновое число Ферми, $p_{\mathrm{F}}=m v_{\mathrm{F}}-$ импульс электрона на поверхности Ферми, $v_{\mathrm{F}}-$ скорость электрона на поверхности Ферми. Тогда

$$
\mathbf{k}_{\perp}^{2} d \mathbf{k}=k_{\mathrm{F}}^{5}\left(K^{2}-K_{x}^{2}\right) d^{3} K=k_{\mathrm{F}}^{5}\left(K_{y}^{2}+K_{z}^{2}\right) d^{3} K=k_{\mathrm{F}}^{5} K_{\perp}^{2} d^{3} K,
$$

где $K_{\perp}^{2}=K^{2}-K_{x}^{2}=K_{y}^{2}+K_{z}^{2}$. Энергию $E_{\mathbf{k}}$ выразим через энергию Ферми $E_{\mathrm{F}}=$ $m v_{\mathrm{F}}^{2} / 2$. Имеем

$$
E_{\mathbf{k}}=\frac{\hbar^{2} \mathbf{k}^{2}}{2 m}=\frac{\hbar^{2} k_{\mathrm{F}}^{2}}{2 m} \mathbf{K}^{2}=\frac{p_{\mathrm{F}}^{2}}{2 m} \mathbf{K}^{2}=E_{\mathrm{F}} \mathbf{K}^{2} \equiv E_{\mathbf{K}}, \quad E_{\mathbf{k}-\mathbf{q}}=\frac{\hbar^{2} k_{\mathrm{F}}^{2}}{2 m}\left(\mathbf{K}-\frac{\mathbf{q}}{k_{\mathrm{F}}}\right)^{2} .
$$


Далее обозначим волновой вектор $\mathbf{q}$ через $\mathbf{k}$ и введем безразмерный волновой вектор $\mathbf{q}=\mathbf{k} / k_{\mathrm{F}}$. Тогда

$$
E_{\mathbf{k}-\mathbf{q}}=\frac{\hbar^{2} k_{\mathrm{F}}^{2}}{2 m}(\mathbf{K}-\mathbf{q})^{2}=E_{\mathrm{F}}(\mathbf{K}-\mathbf{q})^{2}=E_{\mathbf{K}-\mathbf{q}}
$$

Следовательно, с учетом последних равенств формулы для интегралов $J_{\omega}$ и $J_{\nu}$ можно переписать в виде

$$
\begin{aligned}
J_{\nu} & =\frac{\hbar^{2} k_{\mathrm{F}}^{5}}{8 \pi^{3} m N} \int \frac{f_{\mathbf{K}}-f_{\mathbf{K}-\mathbf{q}}}{E_{\mathbf{K}}-E_{\mathbf{K}-\mathbf{q}}} K_{\perp}^{2} d^{3} K, \\
J_{\omega} & =\frac{\hbar^{2} k_{\mathrm{F}}^{5}}{8 \pi^{3} m N} \int \frac{f_{\mathbf{K}}-f_{\mathbf{K}-\mathbf{q}}}{E_{\mathbf{K}}-E_{\mathbf{K}-\mathbf{q}}-\hbar(\omega+i \nu)} K_{\perp}^{2} d^{3} K,
\end{aligned}
$$

где $f_{\mathbf{K}}=\left(1+e^{\left(E_{\mathbf{K}}-\mu\right) / k_{\mathrm{B}} T}\right)^{-1}$.

\section{5. ВЫРОЖДДННАЯ ПЛАЗМА}

Далее мы будем рассматривать случай вырожденной плазмы. В этом случае

$$
f_{\mathbf{K}}=\Theta_{\mathbf{K}} \equiv \Theta\left(E_{\mathbf{F}}-E_{\mathbf{K}}\right)=\Theta\left(1-\mathbf{K}^{2}\right), \quad \Theta_{\mathbf{K}-\mathbf{q}}=\Theta\left(1-(\mathbf{K}-\mathbf{q})^{2}\right),
$$

где $\Theta(x)$ - функция Хевисайда. С учетом этих соотношений выражения для интегралов $J_{\omega}$ и $J_{\nu}$ преобразуются к виду

$$
\begin{aligned}
J_{\omega} & =\frac{3 E_{\mathrm{F}}}{4 \pi} \int \frac{\Theta_{\mathbf{K}}-E_{\mathbf{K}-\mathbf{q}}}{E_{\mathbf{K}}-E_{\mathbf{K}-\mathbf{q}}-\hbar(\omega+i \nu)} K_{\perp}^{2} d^{3} K, \\
J_{\nu} & =\frac{3 E_{\mathrm{F}}}{4 \pi} \int \frac{\Theta_{\mathbf{K}}-E_{\mathbf{K}-\mathbf{q}}}{E_{\mathbf{K}}-E_{\mathbf{K}-\mathbf{q}}} K_{\perp}^{2} d^{3} K .
\end{aligned}
$$

Вычисление поперечной электрической проводимости и диэлектрической проницаемости проведем по формулам (4.3). Вычислим интегралы $J_{\omega}$ и $J_{\nu}$. Заметим, что

$$
E_{\mathbf{K}}-E_{\mathbf{K}-\mathbf{q}}=\hbar v_{\mathrm{F}} k\left(K_{x}-\frac{q}{2}\right), \quad E_{\mathbf{K}}-E_{\mathbf{K}-\mathbf{q}}-\hbar(\omega+i \nu)=\hbar v_{\mathrm{F}} k\left(K_{x}-\frac{q}{2}-\frac{z}{q}\right),
$$

где

$$
z=x+i y, \quad x=\frac{\omega}{k_{\mathrm{F}} v_{\mathrm{F}}}=\frac{\hbar \omega}{2 E_{\mathrm{F}}}, \quad y=\frac{\nu}{k_{\mathrm{F}} v_{\mathrm{F}}}=\frac{\hbar \nu}{2 E_{\mathrm{F}}}, \quad q=\frac{k}{k_{\mathrm{F}}} .
$$

С помощью соотношений (5.1) для интегралов $J_{\omega}$ и $J_{\nu}$ получаем следующие выражения:

$$
J_{\omega}=\frac{3}{8 \pi q} \int \frac{\Theta_{\mathbf{K}}-\Theta_{\mathbf{K}-\mathbf{q}}}{K_{x}-q / 2-z / q} K_{\perp}^{2} d^{3} K, \quad J_{\nu}=\frac{3}{8 \pi q} \int \frac{\Theta_{\mathbf{K}}-\Theta_{\mathbf{K}-\mathbf{q}}}{K_{x}-q / 2} K_{\perp}^{2} d^{3} K .
$$

Преобразуем первый интеграл:

$$
J_{\omega}=\frac{3}{8 \pi q} \int \frac{\Theta\left(1-\mathbf{K}^{2}\right)-\Theta(1-(\mathbf{K}-\mathbf{q}))^{2}}{K_{x}-z / q-q / 2} K_{\perp}^{2} d^{3} K=\frac{3}{16} \int_{-1}^{1} \frac{\left(1-t^{2}\right)^{2}}{(t-z / q)^{2}-q^{2} / 4} d t .
$$


Аналогично второй интеграл можно представить в виде

$$
J_{\nu}=\frac{3}{8 \pi q} \int \frac{\Theta_{\mathbf{K}}-\Theta_{\mathbf{K}-\mathbf{q}}}{K_{x}-q / 2} K_{\perp}^{2} d^{3} K=\frac{3}{16} \int_{-1}^{1} \frac{\left(1-t^{2}\right)^{2}}{t^{2}-q^{2} / 4} d t=\frac{3}{8} \int_{0}^{1} \frac{\left(1-t^{2}\right)^{2}}{t^{2}-q^{2} / 4} d t .
$$

Пусть $x_{\mathrm{p}}=\omega_{\mathrm{p}} / k_{\mathrm{F}} v_{\mathrm{F}}=\hbar \omega_{\mathrm{p}} / 2 E_{\mathrm{F}}$ - безразмерная плазменная частота. Вернемся к формулам (4.3) и представим их в безразмерных параметрах:

$$
\frac{\sigma_{\mathrm{tr}}}{\sigma_{0}}=\frac{i y}{x}\left(1+\frac{x J_{\omega}+i y J_{\nu}}{x+i y}\right), \quad \varepsilon_{\mathrm{tr}}=1-\frac{x_{\mathrm{p}}^{2}}{x^{2}}\left(1+\frac{x J_{\omega}+i y J_{\nu}}{x+i y}\right) .
$$

Перепишем эти соотношения как

$$
\frac{\sigma_{\mathrm{tr}}}{\sigma_{0}}=\frac{i y}{x}\left(1+\frac{3 x T_{1}(q, z)+3 i y T_{0}(q)}{16 z}\right), \quad \varepsilon_{\mathrm{tr}}=1-\frac{x_{\mathrm{p}}^{2}}{x^{2}}\left(1+\frac{3 x T_{1}(q, z)+3 i y T_{0}(q)}{16 z}\right),
$$

где $T_{0}(q)=2 T_{1}(q, 0)$ и

$$
\begin{aligned}
T_{1}(q, z) & =\int_{-1}^{1} \frac{\left(1-t^{2}\right)^{2}}{(t-z / q)^{2}-q^{2} / 4} d t= \\
= & -\frac{10}{3}+\frac{4 z^{2}}{q^{2}}+\frac{q^{2}}{2}+\frac{1}{q}\left[\left(1-\frac{z^{2}}{q^{2}}\right)^{2}+\frac{q^{4}}{16}-\frac{q^{2}}{2}+\frac{3 z^{2}}{2}\right] \ln \frac{(1-q / 2)^{2}-z^{2} / q^{2}}{(1+q / 2)^{2}-z^{2} / q^{2}}- \\
& -\frac{z q}{2}\left[1+\frac{4}{q^{2}}\left(1-\frac{z^{2}}{q^{2}}\right)\right] \ln \frac{(1-z / q)^{2}-q^{2} / 4}{(1+z / q)^{2}-q^{2} / 4} .
\end{aligned}
$$

Для сравнения представим формулу Линдхарда [2] в наших обозначениях следующим образом:

$$
\frac{\sigma_{\mathrm{tr}}^{\mathrm{Lin}}}{\sigma_{0}}=\frac{i y}{x}+\frac{3 i y}{16 x} \int_{-1}^{1} \frac{\left(1-t^{2}\right)^{2}}{(t-z / q)^{2}-q^{2} / 4} d t
$$

Из соотношений (5.2) и (5.3) видно, что мнимые части поперечных электрических проводимостей из настоящей работы и из работы Линдхарда при $y \rightarrow 0$ совпадают.

Представим формулу для поперечной электрической проводимости в явном виде:

$$
\begin{aligned}
\frac{\sigma_{\mathrm{tr}}}{\sigma_{0}}= & \frac{i y}{x}\left\{1+\frac{3 i y}{8 x}\left(-\frac{5}{3}+\frac{q^{2}}{4}+\frac{\left(q^{2}-4\right)^{2}}{16 q} \ln \frac{2-q}{2+q}\right)+\frac{3 x}{16 z}\left[-\frac{10}{3}+\frac{4 z^{2}}{q^{2}}+\frac{q^{2}}{2}+\right.\right. \\
& +\frac{1}{q}\left(\left(1-\frac{z^{2}}{q^{2}}\right)^{2}+\frac{q^{4}}{16}-\frac{q^{2}}{2}+\frac{3 z^{2}}{2}\right) \ln \frac{(1-q / 2)^{2}-z^{2} / q^{2}}{(1+q / 2)^{2}-z^{2} / q^{2}}- \\
& \left.\left.-\frac{z q}{2}\left(1+\frac{4}{q^{2}}\left(1-\frac{z^{2}}{q^{2}}\right)\right) \ln \frac{(1-z / q)^{2}-q^{2} / 4}{(1+z / q)^{2}-q^{2} / 4}\right]\right\} .
\end{aligned}
$$

\section{6. ЧАСТНЫЕ СЛУЧАИ ЭЛЕКТРИЧЕСКОЙ ПРОВОДИМОСТИ}

Исследуем частные случаи электрической проводимости. Рассмотрим формулу (4.3) и преобразуем ее к виду

$$
\frac{\sigma_{\mathrm{tr}}}{\sigma_{0}}=\frac{i \nu}{\omega}\left[1+\frac{3}{8 \pi(\omega+i \nu) q} \int\left(\frac{i \nu}{K_{x}-q / 2}+\frac{\omega}{K_{x}-q / 2-z / q}\right)\left(\Theta_{\mathbf{K}}-\Theta_{\mathbf{K}-\mathbf{q}}\right) K_{\perp}^{2} d^{3} K\right] .
$$



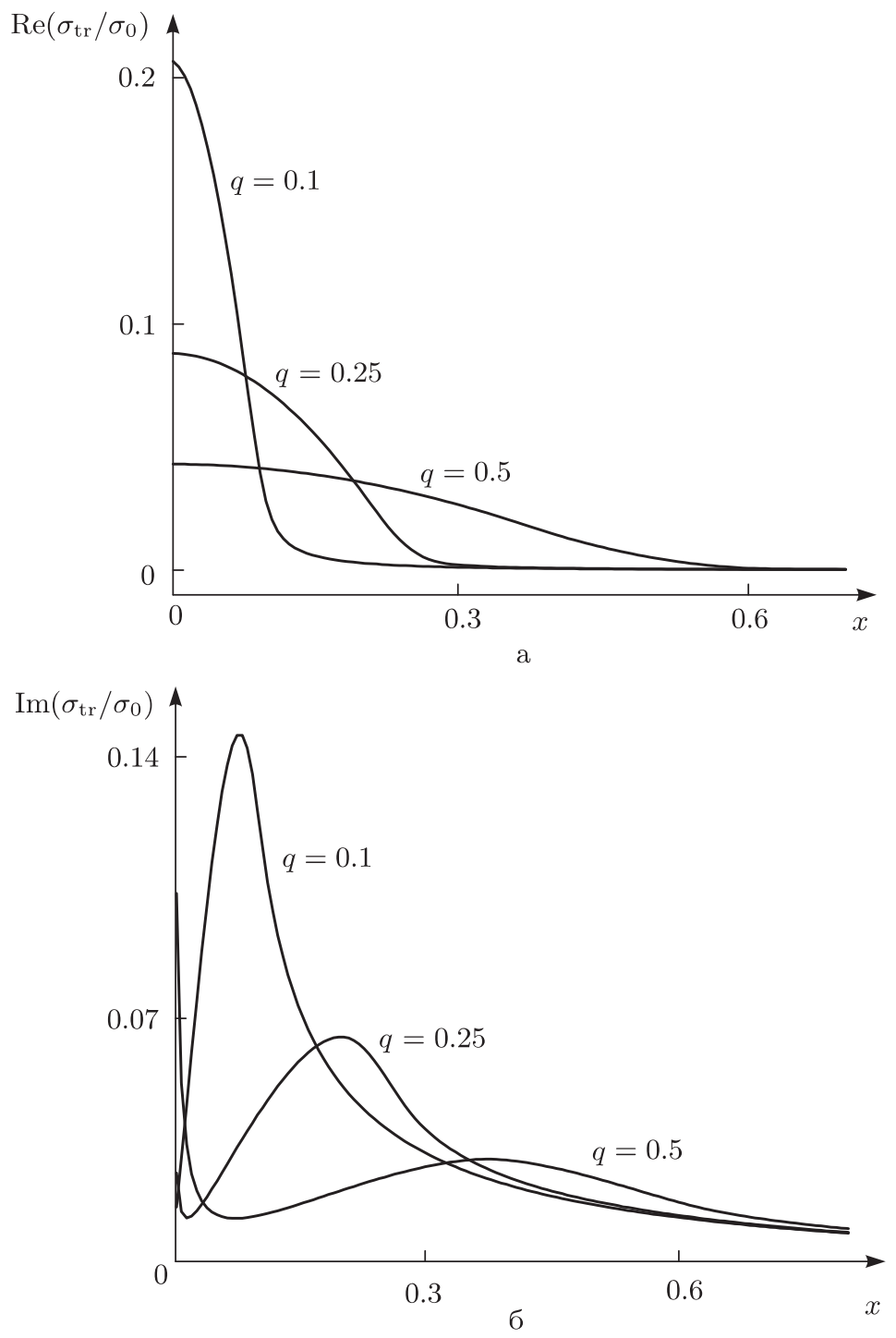

Рис. 1. Действительная (а) и мнимая (б) части поперечной электрической проводимости как функции безразмерной частоты колебаний $x$ при $y=0.01$ и различных значениях безразмерного волнового числа $q$.

Подынтегральные выражения содержат функцию $\varphi(\mathbf{q})=\Theta\left(1-K^{2}\right)-\Theta\left(1-(\mathbf{K}-\mathbf{q})^{2}\right)$. В линейном приближении мы получаем

$$
\varphi(\mathbf{q})=-2 \delta\left(1-K^{2}\right) K_{x} q=-\delta(1-K) K_{x} q
$$

Заметим, что

$$
\frac{3}{8 \pi} \int \delta(1-K) K_{\perp}^{2} d^{3} K=1
$$




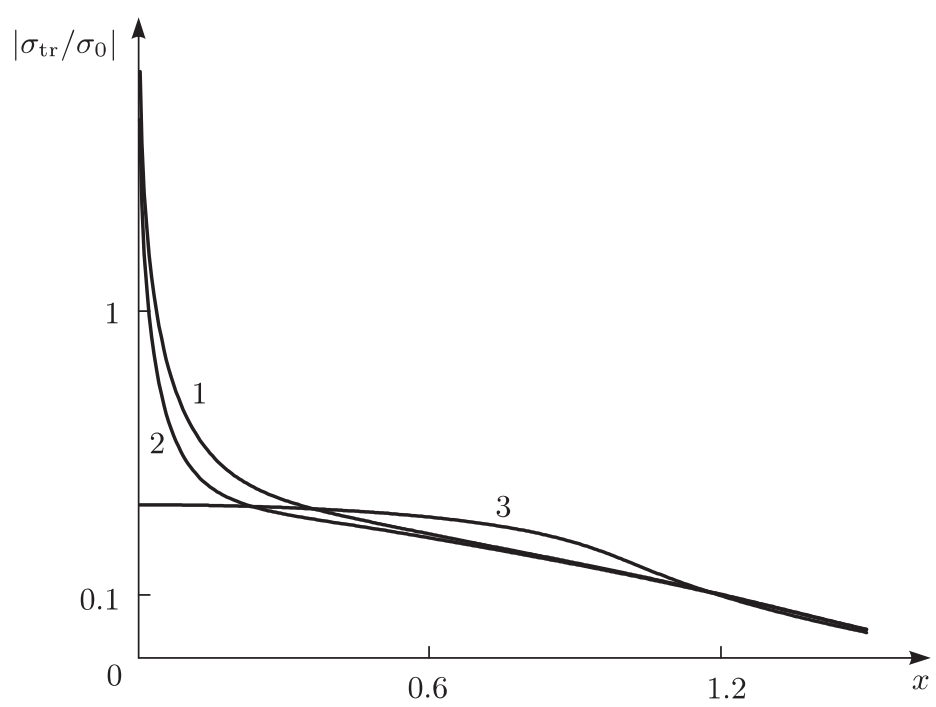

Рис. 2. Зависимость модуля отношения $\sigma_{\mathrm{tr}} / \sigma_{0}$ от безразмерной частоты колебаний плазмы (кривая 1) в сравнении с аналогичной зависимостью проводимости по Линдхарду (кривая 2) и классической проводимости (кривая 3 ) при $y=0.1, q=1$.

следовательно, выражение для электрической проводимости можно преобразовать к виду

$$
\begin{aligned}
\frac{\sigma_{\mathrm{tr}}}{\sigma_{0}}= & \frac{i \nu}{\omega}\left[\frac{\omega}{\omega+i \nu}-\frac{3 i \nu q}{16 \pi(\omega+i \nu)} \int \frac{\delta(1-K)}{K_{x}-q / 2} K_{\perp}^{2} d^{3} K-\right. \\
& \left.-\frac{3 \omega}{8 \pi(\omega+i \nu)} \int \frac{K_{x} \delta(1-K)}{K_{x}-z / q-q / 2} K_{\perp}^{2} d^{3} K\right] .
\end{aligned}
$$

Видно, что $\sigma_{\mathrm{tr}} \rightarrow \sigma_{0} i \nu /(\omega+i \nu)$, при $q \rightarrow 0$ и при $\omega=0$ мы получаем в точности статическую проводимость: $\sigma_{\mathrm{tr}}=\sigma_{0}$.

Покажем теперь, что при малых $q$ формула (6.2) приводит к известному выражению для проводимости вырожденной фермиевской плазмы. В самом деле, заметим, что при малых $q$ первый интеграл из (6.2) пропорционален $q^{2}$. Отбросим этот интеграл. В знаменателе второго интеграла пренебрежем членом $q / 2$, ибо $q / 2 \ll|z| / q$. В результате для малых $q$ получаем

$$
\frac{\sigma_{\mathrm{tr}}}{\sigma_{0}}=\frac{i \nu}{\omega+i \nu}\left[1-\frac{3}{8 \pi} \int \frac{\delta(1-K) K_{x}}{K_{x}-z / q} K_{\perp}^{2} d^{3} K\right] .
$$

Пользуясь снова равенством (6.1), на основании (6.3) получаем соотношение

$$
\frac{\sigma_{\mathrm{tr}}}{\sigma_{0}}=-\frac{3 y}{8 \pi q} \int \frac{\delta(1-K)}{K_{x}-q / 2} K_{\perp}^{2} d^{3} K
$$


которое приводит к известному выражению для электрической проводимости в вырожденной фермиевской плазме:

$$
\sigma_{\mathrm{tr}}^{\text {classic }}=-\frac{3 i y \sigma_{0}}{4 q} \int_{-1}^{1} \frac{1-\mu^{2}}{\mu-z} d \mu=\frac{3 i y \sigma_{0}}{4 q}\left[\frac{2 z}{q}+\left(z^{2}-q^{2}\right) \ln \frac{z-q}{z+q}\right] .
$$

На рис. 1 представлены рассчитанные по формулам настоящей работы зависимости действительной и мнимой частей поперечной электрической проводимости от безразмерной частоты колебаний $x$ при $y=0.01$. Отметим, что, например, для калия [3] $\omega_{\mathrm{p}}=6.5 \cdot 10^{15} \mathrm{c}^{-1}, v_{\mathrm{F}}=8.52 \cdot 10^{7} \mathrm{~cm} \cdot \mathrm{c}^{-1}$, а значению $y=0.01$ отвечает частота $\nu=6.3 \cdot 10^{13} \mathrm{c}^{-1}$. Различные кривые на рис. 1 отвечают значениям безразмерного волнового числа $q=0.1,0.25,0.5$. Видно, что действительная часть является монотонно убывающей функцией, а мнимая часть имеет максимум, величина которого убывает с ростом волнового числа. С ростом частоты колебаний плазмы и действительная, и мнимая части убывают до нуля.

На рис. 2 показана зависимость модуля отношения $\sigma_{\mathrm{tr}} / \sigma_{0}$ от безразмерной частоты колебаний плазмы, рассчитанная по формулам настоящей работы (кривая 1), в сравнении с аналогичными зависимостями для проводимости по Линдхарду (кривая 2) и классической проводимости (кривая 3); для всех кривых на рис. 2 взяты $y=0.1, q=1$. Из рис. 2 видно, что при $x \rightarrow 0$ проводимость по Линдхарду совпадает с проводимостью квантовой плазмы, а при $x \rightarrow \infty$ совпадают все три проводимости.

Рис. 3 позволяет сравнить зависимости действительной части и мнимой части отношения $\sigma_{\mathrm{tr}} / \sigma_{0}$ от безразмерного волнового числа $q$ : приведены результаты расчета по нашим формулам (кривые 1), по формулам Линдхарда (кривые 2) и по формулам для классической проводимости (кривые 3). Из рис. 3 видно, что при малых значениях частоты столкновений проводимость по Линдхарду практически совпадает с проводимостью квантовой плазмы при малых значениях волнового числа.

\section{7. ЗАКЛЮЧЕНИЕ}

Выведена формула для электрической проводимости в квантовой столкновительной плазме. Для этой цели используется кинетическое уравнение с интегралом столкновений в форме релаксационной модели в пространстве импульсов. Исследованы различные частные случаи. Выделен и исследован случай вырожденной фермиевской плазмы. Проведено сравнение графиков проводимости, полученных по формулам из настоящей работы, с проводимостью по Линдхарду и с классической проводимостью.

\section{Список литературы}

[1] N. D. Mermin, Phys. Rev. B, 1:5 (1970), 2362-2363.

[2] J. Lindhard, Danske Vid. Selsk. Mat.-Fys. Medd., 28:8 (1954), 1-57.

[3] K. L. Kliewer, R. Fuchs, Phys. Rev., 181:2 (1969), 552-558.

[4] M. Dressel, G. Grüner, Electrodynamics of Solids. Optical Properties of Electrons in Matter, Cambridge Univ. Press, Cambridge, 2003.

[5] A. P. van Gelder, Phys. Rev., 187:3 (1969), 833-842.

[6] D. C. Mattis, J. Bardeen, Phys. Rev., 111:2 (1958), 412-417.

[7] Н. А. Зимбовская, УФН, 181:8 (2011), 793-826. 
[8] W. E. Jones, K. L. Kliewer, R. Fuchs, Phys. Rev., 178:3 (1969), 1201-1203.

[9] J. M. Pitarke, V. M. Silkin, E. V. Chulkov, P. M. Echenique, Rep. Prog. Phys., 70:1 (2007), 1-87, arXiv: cond-mat/0611257.

[10] J. E. Valdes, P. Vargas, N. R. Arista, Nucl. Instrum. Meth. Phys. Res. B, 174:1-2 (2001), $9-15$.

[11] D. Anderson, B. Hall, M. Lisak, M. Marklund, Phys. Rev. E, 65:4 (2002), 046417, 5 pp., arXiv: quant-ph/0305102.

[12] П. К. Шукла, Б. Элиассон, УФН, 180:1 (2010), 55-82.

[13] B. Eliasson, P. K. Shukla, J. Plasma Phys., 76:1 (2010), 7-17, arXiv: 0911.4594.

[14] A. Wierling, Interpolation between static local field corrections and the Drude model by a generalized Mermin approach, arXiv: 0812.3835.

[15] G. Brodin, M. Marklund, G. Manfredi, Phys. Rev. Lett., 100:17 (2008), 175001, 4 pp., arXiv: 0802.0169.

[16] G. Manfredi, F. Haas, Phys. Rev. B, 64:7 (2001), 075316, 7 pp., arXiv: cond-mat/0203394.

[17] G. Manfredi, "How to model quantum plasmas", Topics in Kinetic Theory (Toronto, Canada, March 24-26, 2004), Fields Institute Communications, 46, eds. T. Passot, C. Sulem, P. L. Sulem, AMS, Providence, RI, 2005, 263-287, arXiv: quant-ph/0505004.

[18] H. Reinholz, G. Röpke, Phys. Rev. E, 85:3 (2012), 036401, 16 pp.

[19] D. Peins, P. Nozières, The Theory of Quantum Liquids, v. 1: Normal Fermi Liquids, Benjamin, New York, Amsterdam, 1966.

[20] P. C. Martin, Phys. Rev., 161:1 (1967), 143-155.

Поступила в редакцию 8.10.2012, после доработки 11.11.2012 
ПОПЕРЕЧНАЯ ЭЛЕКТРИЧЕСКАЯ ПРОВОДИМОСТЬ КВАНТОВОЙ ПЛАЗМЫ 